\title{
On Delay Independent Stabilization Analysis for a Class of Switched Large-Scale Time-Delay Systems
}

\author{
Chi-Jo Wang and Juing-Shian Chiou \\ Department of Electrical Engineering, Southern Taiwan University of Science and Technology, Tainan City, Taiwan \\ Correspondence should be addressed to Juing-Shian Chiou; jschiou@mail.stust.edu.tw
}

Received 27 July 2013; Accepted 24 November 2013

Academic Editor: Shengqiang Liu

Copyright ( 2013 C.-J. Wang and J.-S. Chiou. This is an open access article distributed under the Creative Commons Attribution License, which permits unrestricted use, distribution, and reproduction in any medium, provided the original work is properly cited.

In view of the state-driven switching method, the sufficient stability conditions with delay independence will be derived for the switched large-scale time-delay systems. A new stability criterion of switched large-scale time-delay systems is deduced by Lyapunov stability theorem. The method can be applied to cases when all individual switched systems are unstable. Finally, one example is exploited to illustrate the proposed schemes.

\section{Introduction}

Switched systems form an important class of hybrid systems. Such systems can be described by a family of continuoustime subsystems (or discrete-time subsystems) and a rule that orchestrates the switching among them. It is well known that a wide class of physical systems in power systems, chemical procedure control systems, navigation systems, automobile speed change systems, and so forth may be appropriately described by the switched model [1-10]. In the study of switched systems, most works have been centered on the problem of stability. In the last two decades, there have been increasing interests in the stability analysis for such switched systems; see, for example, [11] and the references cited therein.

Two important methods are used to construct the switching law for the stability analysis of the switched systems. One is the state-driven switching strategy; the other is the timedriven switching strategy. The time-driven switching method is formed based on the main concept of a dwell time. If at least one stable subsystem exits, then the switched system is stable with a proper dwell time switching law. However, in reality, it is not unusual to encounter cases in which all subsystems are unstable. Therefore, [6] proposed that all subsystem can be unstable for time-driven switching method. We would have to turn to the state-driven switching strategy, from which many choices of switching laws ensuring stability exist, even if all the subsystems are unstable. Reference [5] proposed quadratic stabilizability via state feedback for both continuous-time and discrete-time switched linear systems that are composed of polytopic uncertain subsystems. For continuous-time switched linear systems, if there exists a common positive definite matrix for stability of all convex combinations of the extreme points which belong to different subsystem matrices, then the switched system is quadratically stabilizable via state feedback. The switching rules can be obtained by using the obtained common positive definite matrix.

Furthermore, the time-delay phenomenon is also unavoidable in practical systems, for instance, chemical process, long distance transmission line, hybrid procedure, electron network, and so forth. Time delays may cause instability and poor performance for practical systems [12]. In view of the aforementioned facts, the stability of switched systems with time delay is very worthy of research $[13,14]$. Reference [13] studied stability and $L_{2}$ gain properties for a class of switched systems which are composed of a finite number of linear time-invariant symmetric systems with time delay and showed that, when all subsystems are in sense of satisfying an LMI, the switched system is asymptotically stable under arbitrary switching. Reference [14] proposed a delay-dependent robust $H_{\infty}$ controller design method for the uncertain linear switched systems with time delay. Reference 
[15] investigated the problem of robust $L_{\infty}$ reliable control for uncertain switched nonlinear systems with time delay and actuator failures. References $[16,17]$ concerned with the problem of delay-dependent stability analysis for a class of two-dimensional discrete switched systems described by the Roesser model with state delays. Besides, a large-scale system is often considered as a set of interconnected subsystems. The advantage of this aspect in stability analysis is to reduce complexity $[18,19]$. Recently, many approaches have been used to investigate the stability and stabilization of large-scale time-delay systems $[20,21]$. Therefore, the stability analysis of switched large-scale time-delay systems is very worthy to be researched.

Basically, current efforts to achieve stability in timedelay systems can be divided into two categories, namely, delay-independent criteria and delay-dependent criteria. For the delay-dependent criteria, the Lyapunov-Krasovskii functional technique is most suitable for application; the proposed criterion adopting this technique performs much better than several existing criteria [22-24]. In this paper, the delayindependent stability of switched large-scale time-delay system is considered. In view of the state-driven switching method, a new stability criterion of switched large-scale timedelay system is deduced by Lyapunov stability theorem [25]. This method can be applied to cases when all individual switched systems are unstable.

The following notations will be used throughout the paper: $\lambda(A)$ stands for the eigenvalues of matrix $A, \lambda_{\max }(A)$ means the maximum eigenvalue in matrix $A$; that is, $\lambda_{\text {max }}(A)=\operatorname{Max}[\lambda(A)],\|A\|$ denotes the norm of matrix $A$; that is, $\|A\|=\operatorname{Max}\left[\lambda\left(A^{T} A\right)\right]^{1 / 2}$, and $A^{T}$ is the transpose of matrix $A$.

\section{System Description and Problem Statement}

Consider the switched time-delay large-scale systems

$$
\begin{gathered}
\dot{x}_{i}(t)=A_{\sigma(x) \_i i} x_{i}(t)+\sum_{\substack{j \neq i \\
j=1}}^{N} A_{\sigma(x) \_j} x_{j}(t)+\sum_{j=1}^{N} B_{\sigma(x) i j} x_{j}(t-\tau) \\
x_{i}(t)=\psi_{i}(t), \quad t \in[-\tau, 0],
\end{gathered}
$$

where $x_{i}(t) \in R^{n_{i}}$ are the state vectors of the $i$ th subsystem, $i=1,2, \ldots, N, n=\sum_{i=1}^{N} n_{i}, A_{\sigma(x) \_i i}, A_{\sigma(x) i j}$, and $B_{\sigma(x)_{-} i j}$ are some constant matrices of compatible dimensions, $x(t)=\left[\begin{array}{llll}x_{1}^{T}(t) & x_{2}^{T}(t) & \cdots & x_{N}^{T}(t)\end{array}\right]^{T}$, and $\sigma(x(t)): R^{n}$ $\rightarrow\{1,2, \ldots, r\}$ is a piecewise constant function of time, called a switch signal; that is, the matrix $A_{\sigma(x) \_i i}$ switches between matrices $A_{1 i i}, A_{2 i i}, \ldots, A_{r_{i i}}$ belonging to the set $A \equiv\left\{A_{1 \_11}, A_{2 i i}, \ldots, A_{r_{i} i}\right\}$ and $A_{l i i} l \in\{1,2, \ldots, r\}$. The matrix $B_{\sigma(x)_{-} i j}$ switches between matrices $B_{1_{-} i j}, B_{2 \_i j}, \ldots, B_{r_{-} i j}$ belonging to the set $B \equiv\left\{B_{1_{-i j}}, B_{2 i j}, \ldots, B_{r_{-} i j}\right\}$ and $B_{l i j}, i, j \in$ $\{1,2, \ldots, N\} . A_{\sigma(x)_{i} i i}, A_{\sigma(x) \_j}$, and $B_{\sigma(x)_{-} i j}$ are matrices of compatible dimensions. $\tau>0$ is the time-delay duration. $\psi_{i}(t)$ is a vector-valued initial continuous function defined on the interval $[-\tau, 0]$, and finally $\psi_{i}(t)$, defined on $-\tau \leq t \leq 0$, is the initial condition of the state, $N$ is the number of interconnected subsystems, $n$ is the whole systems dimension, and $r$ is the number of individual systems.

As we have indicated, very few research articles have ever discussed the stability of the switched time-delay large-scale system (1a) and (1b). To facilitate our analysis later, some helpful lemmas are given below first.

First, we consider the normal time-delay system

$$
\dot{x}(t)=A x(t)+B x(t-\tau),
$$

where $x \in R^{n}, A$ and $B$ are matrices in proper dimensions, and $\tau$ is the delay duration.

Lemma 1 (see [26]). The stability with delay independence of system (2) implies the stability with delay independence for the following systems:

$$
\dot{w}(t)=(A+z B) w(t), \quad \forall|z| \leq 1,
$$

and vice versa.

Lemma 2. There exists a switching law for the switched system (1a) and (1b) such that the system is asymptotically stable if there exist positive constants $\alpha_{i}(1 \leq i \leq N)$ satisfying $\sum_{i=1}^{N} \alpha_{i}=1$ such that the convex combination of the whole switched time-delay system

$$
\dot{x}(t)=\sum_{i=1}^{N} \alpha_{i}\left[A_{i} x(t)+B_{i} x(t-\tau)\right]
$$

is an asymptotically stable system.

Proof. Since there exist positive numbers $\alpha_{i}(1 \leq i \leq N)$ such that $\dot{x}(t)=\sum_{i=1}^{N} \alpha_{i}\left[A_{i} x(t)+B_{i} x(t-\tau)\right]$ is asymptotically stable, there exists a Lyapunov function $V(x)$ such that

$$
\frac{\partial V}{\partial x} \sum_{i=1}^{N} \alpha_{i}\left[A_{i} x(t)+B_{i} x(t-\tau)\right]<0 .
$$

It follows that, for any $t$, there exists an $i \in\{1,2, \ldots, N\}$ such that

$$
\frac{\partial V}{\partial x}\left(A_{i} x(t)+B_{i} x(t-\tau)\right)<0 .
$$

From (6), it implies that a convex combination of the corresponding Lyapunov function is negative along the trajectory and from, at least one must be negative. Thus, switched timedelay system (la) and (1b) is asymptotically stable.

Remark 3. Condition (6) will be used to design switching laws. The technique is that the switching is forced so that the active system is always in the region corresponding to the Lyapunov function with negative derivative. 
Lemma 4. In view of Lemma 1 , the stability with delay independence of $\dot{x}(t)=\sum_{i=1}^{N} \alpha_{i}\left[A_{i} x(t)+B_{i} x(t-\tau)\right]$ implies the stability with delay independence for the following systems:

$$
\dot{w}(t)=\sum_{i=1}^{N} \alpha_{i}\left[A_{i}+z B_{i}\right] w(t), \quad \forall|z| \leq 1,
$$

and vice versa.

Thus, the system (7) is an asymptotically stable system; then, the system (4) is stable. That is, the switched time-delay system (1a) and (1b) is also stable.

A sufficient condition for stability of the switched timedelay large-scale system (1a) and (1b) is established in the following theorem by using the state-switched method.

\section{Stabilization Analysis of Switched Large-Scale Time-Delay System}

The system (1a) and (1b) can be rewritten as the following:

$$
\dot{X}(t)=A_{l} X+B_{l} X(t-\tau),
$$

where

$$
\begin{aligned}
& A_{l}=\left[\begin{array}{cccc}
A_{l_{-11}} & A_{l_{-12}} & \cdots & A_{l_{-1 N}} \\
A_{l_{-21}} & A_{l \_1} & \cdots & A_{l_{-2 N}} \\
\vdots & \vdots & \vdots & \vdots \\
A_{l_{\perp N 1}} & A_{l_{-N 2}} & \cdots & A_{l_{\perp N N}}
\end{array}\right], \\
& B_{l}=\left[\begin{array}{cccc}
B_{l_{-11}} & B_{l_{-} 12} & \cdots & B_{l-1 N} \\
B_{l \_21} & B_{l \_21} & \cdots & B_{l \_2 N} \\
\vdots & \vdots & \vdots & \vdots \\
B_{l-N 1} & B_{l \_N 2} & \cdots & B_{l \_N N}
\end{array}\right] \text {, } \\
& X(t)=\left[\begin{array}{llll}
x_{1}^{T} & x_{2}^{T} & \cdots & x_{N}^{T}
\end{array}\right]^{T} .
\end{aligned}
$$

First, we consider the switched large-scale time-delay system (la) and (1b) with $r=N=2$ :

$$
\begin{aligned}
& \dot{x}_{1}(t)=A_{l_{-} 11} x_{1}(t)+A_{l_{-12}} x_{2}(t)+B_{l_{-11}} x_{1}(t-\tau) \\
& +B_{l \_12} x_{2}(t-\tau) \\
& \dot{x}_{2}(t)=A_{l \_21} x_{1}(t)+A_{l \_2} x_{2}(t)+B_{l \_21} x_{1}(t-\tau) \\
& +B_{l \_22} x_{2}(t-\tau) \text {; }
\end{aligned}
$$

that is,

$$
\dot{X}(t)=\left[\begin{array}{ll}
A_{l_{-11}} & A_{l_{-12}} \\
A_{l \_21} & A_{l_{-22}}
\end{array}\right] X(t)+\left[\begin{array}{ll}
B_{l_{-11}} & B_{l_{-12}} \\
B_{l \_21} & B_{l_{-22}}
\end{array}\right] X(t-\tau) .
$$

In the light of Lemma 2, it is obvious that the stability of the switched time-delay system ((10a), (10b), and (10c)) is equivalent to that of the system

$$
\begin{gathered}
\dot{x}_{1}(t)=\sum_{l=1}^{2} \alpha_{l}\left[A_{l_{-} 11} x_{1}(t)+A_{l_{-} 12} x_{2}(t)+B_{l_{-} 11} x_{1}(t-\tau)\right. \\
\left.+B_{l_{\_} 12} x_{2}(t-\tau)\right]
\end{gathered}
$$

$$
\begin{gathered}
\dot{x}_{2}(t)=\sum_{l=1}^{2} \alpha_{l}\left[A_{l \_21} x_{1}(t)+A_{l \_22} x_{2}(t)+B_{l \_21} x_{1}(t-\tau)\right. \\
\left.+B_{l \_22} x_{2}(t-\tau)\right]
\end{gathered}
$$

that is,

$$
\begin{aligned}
\dot{X}(t)= & \sum_{l=1}^{2} \alpha_{l}\left(\left[\begin{array}{ll}
A_{l_{-11}} & A_{l_{-12}} \\
A_{l \_21} & A_{l_{-} 22}
\end{array}\right] X(t)+\left[\begin{array}{ll}
B_{l_{-11}} & B_{l_{-12}} \\
B_{l-21} & B_{l \_22}
\end{array}\right] X(t-\tau)\right) \\
= & \alpha_{1}\left(A_{1} X(t)+B_{1} X(t-\tau)\right) \\
& +\alpha_{2}\left(A_{2} X(t)+B_{2} X(t-\tau)\right) .
\end{aligned}
$$

To investigate the stability of system (11a) and (11b), we choose the Lyapunov function candidate as

$$
V_{i}\left(x_{i}(t)\right)=x_{i}^{T}(t) P_{i} x_{i}(t),
$$

where $P=\operatorname{diag}\left[P_{1}, P_{2}\right]$ is a unique real symmetric positivedefinite matrix satisfying the Lyapunov equations:

$$
A_{i j i}^{T} P_{i}+P_{i} A_{i i i}=-I, \quad i=1,2 .
$$

It will be convenient throughout this section to use the following notations:

$$
\begin{aligned}
S_{1 \_1}= & -\left[\beta P_{\lambda 1}^{2}\left(\left\|B_{1 \_11}\right\|^{2}+\left\|B_{1 \_12}\right\|^{2}\right)\right. \\
& +P_{\lambda 1}+P_{\lambda_{1}}\left\|A_{1 \_12}\right\|+P_{\lambda 2}\left\|A_{1 \_21}\right\| \\
& \left.+\beta P_{\lambda 1} P_{\lambda 2}\left(\left\|B_{1 \_11}\right\|\left\|B_{1 \_21}\right\|+\left\|B_{1 \_12}\right\|\left\|B_{1 \_22}\right\|\right)\right], \\
S_{1 \_2}= & 2 P_{\lambda 1}\left\|A_{2 \_11}\right\|+\beta P_{\lambda 1}^{2}\left(\left\|B_{2 \_11}\right\|^{2}+\left\|B_{2 \_12}\right\|^{2}\right) \\
+ & P_{\lambda 1}+P_{\lambda 2}\left\|A_{2 \_21}\right\|+P_{\lambda 1}\left\|A_{2 \_12}\right\| \\
+ & \beta P_{\lambda 1} P_{\lambda 2}\left(\left\|B_{2 \_11}\right\|\left\|B_{2 \_21}\right\|+\left\|B_{2 \_12}\right\|\left\|B_{2 \_22}\right\|\right) \\
S_{2 \_2}= & 1-\left[\beta P_{\lambda 2}^{2}\left(\left\|B_{2 \_21}\right\|^{2}+\left\|B_{2 \_22}\right\|^{2}\right)+P_{\lambda 2}\right. \\
& +P_{\lambda 2}\left\|A_{2 \_21}\right\|+P_{\lambda 1}\left\|A_{2 \_12}\right\|+\beta P_{\lambda 1} \\
& \left.\times P_{\lambda 2}\left(\left\|B_{2 \_11}\right\|\left\|B_{2 \_1}\right\|+\left\|B_{2 \_12}\right\|\left\|B_{2 \_22}\right\|\right)\right]
\end{aligned}
$$

$$
\begin{aligned}
S_{2 \_1}= & 2 P_{\lambda 2}\left\|A_{1 \_2}\right\|+\beta P_{\lambda 2}^{2}\left(\left\|B_{1 \_21}\right\|^{2}+\left\|B_{1 \_22}\right\|^{2}\right) \\
& +P_{\lambda 2}+P_{\lambda 1}\left\|A_{1 \_12}\right\|+P_{\lambda 2}\left\|A_{1 \_21}\right\| \\
& +\beta P_{\lambda 1} P_{\lambda 2}\left(\left\|B_{1 \_11}\right\|\left\|B_{1 \_21}\right\|+\left\|B_{1 \_12}\right\|\left\|B_{1 \_22}\right\|\right),
\end{aligned}
$$




$$
\begin{gathered}
P_{\lambda 1}=\operatorname{Max}\left(\lambda\left(P_{1}\right)\right), \quad P_{\lambda 2}=\operatorname{Max}\left(\lambda\left(P_{2}\right)\right), \\
\beta=\operatorname{Max}\left(\lambda\left(P_{1}^{-1}\right) \lambda\left(P_{2}^{-1}\right)\right), \quad \underline{\alpha}=\frac{S_{1 \_}}{S_{1 \_1}+S_{1 \_}}, \\
\bar{\alpha}=\frac{S_{2 \_}}{S_{2 \_1}+S_{2 \_}} .
\end{gathered}
$$

Theorem 5. Suppose the switched large-scale time-delay system (1a) and (1b) with $r=N=2$. There exists a switching law such that the switched large-scale time-delay system (1a) and (1b) is asymptotically stable of delay independence, if constant $\alpha_{1} \in(\underline{\alpha}, \bar{\alpha})$ and there matrices $P_{1}, P_{2}>0$ satisfying

$$
\begin{aligned}
& \text { (i) } S_{1 \_1}>0 \\
& \text { (ii) } S_{2 \_-}>0 \\
& \text { (iii) } S_{1 \_1} S_{2 \_2}>S_{1 \_2} S_{2 \_1} \text {. }
\end{aligned}
$$

Proof. The Lyapunov function for the overall system is

$$
V(X(t))=\sum_{i=1}^{2} x_{i}^{T}(t) P_{i} x_{i}(t)=X^{T}(t) P X(t) .
$$

The differential of the Lyapunov function (17) is as follows:

$$
\begin{gathered}
\dot{V}(X(t))=\sum_{l=1}^{2} \alpha_{l}\left[X^{T}(t)\left(A_{l}^{T} P+P A_{l}\right) X(t)+X^{T}(t)\right. \\
\left.\times P B_{l} X(t-\tau)+X^{T}(t-\tau) B_{l}^{T} P X(t)\right] \\
\leq \sum_{l=1}^{2} \alpha_{l}\left[X^{T}(t)\left(A_{l}^{T} P+P A_{l}+P B_{l} P^{-1} B_{l}^{T} P\right) X(t)\right. \\
\left.+X^{T}(t-\tau) P X(t-\tau)\right] \\
\leq \sum_{l=1}^{2} \alpha_{l}\left[X^{T}(t)\left(A_{l}^{T} P+P A_{l}+P B_{l} P^{-1} B_{l}^{T} P\right) X(t)\right. \\
+V(X(t-\tau))] .
\end{gathered}
$$

By using Razumikhin theorem [27], we assume that there exists a real $v>1$ such that

$$
V(X(t-\theta))<v V(X(t)), \quad \text { for } \theta \in[0, \tau] ;
$$

then,

$$
\begin{aligned}
\dot{V}(X(t))<\sum_{l=1}^{2} \alpha_{l}[ & X^{T}(t)\left(A_{l}^{T} P+P A_{l}+P B_{l} P^{-1} B_{l}^{T} P\right) X(t) \\
+ & v V(X(t))] .
\end{aligned}
$$

By continuity, there exists a $v=1+\delta$ with $\delta>0$ sufficiently small such that $\dot{V}(X(t))<0$ for all $i$. Therefore,

$$
\begin{aligned}
& \dot{V}(X(t))<\sum_{l=1}^{2} \alpha_{l}\left[X^{T}(t)\right.\left(A_{l}^{T} P+P A_{l}\right. \\
&+\left.\left.P B_{l} P^{-1} B_{l}^{T} P+P\right) X(t)\right] \\
&<\alpha_{1} X^{T}(t)\left(\left[\begin{array}{cc}
A_{1 \_11} & A_{1 \_12} \\
A_{1 \_21} & A_{1 \_22}
\end{array}\right]^{T}\left[\begin{array}{cc}
P_{1} & 0 \\
0 & P_{2}
\end{array}\right]\right. \\
&+ {\left[\begin{array}{cc}
P_{1} & 0 \\
0 & P_{2}
\end{array}\right]\left[\begin{array}{cc}
A_{1 \_11} & A_{1 \_12} \\
A_{1 \_21} & A_{1.22}
\end{array}\right] } \\
&+\beta\left[\begin{array}{cc}
P_{1} & 0 \\
0 & P_{2}
\end{array}\right]\left[\begin{array}{ll}
B_{1 \_11} & B_{1 \_12} \\
B_{1 \_21} & B_{1.22}
\end{array}\right] \\
& \times\left[\begin{array}{cc}
B_{1 \_11} & B_{1.12} \\
B_{1.21} & B_{1.22}
\end{array}\right]^{T}\left[\begin{array}{cc}
P_{1} & 0 \\
0 & P_{2}
\end{array}\right] \\
&\left.+\left[\begin{array}{cc}
P_{1} & 0 \\
0 & P_{2}
\end{array}\right]\right) X(t)
\end{aligned}
$$

$$
+\alpha_{2} X^{T}(t)\left(\left[\begin{array}{ll}
A_{2 \_11} & A_{2 \_12} \\
A_{2 \_21} & A_{2 \_22}
\end{array}\right]^{T}\left[\begin{array}{cc}
P_{1} & 0 \\
0 & P_{2}
\end{array}\right]\right.
$$$$
+\left[\begin{array}{cc}
P_{1} & 0 \\
0 & P_{2}
\end{array}\right]\left[\begin{array}{ll}
A_{2 \_11} & A_{2-12} \\
A_{2-21} & A_{2-22}
\end{array}\right]
$$$$
+\beta\left[\begin{array}{cc}
P_{1} & 0 \\
0 & P_{2}
\end{array}\right]\left[\begin{array}{ll}
B_{2-11} & B_{2 \_12} \\
B_{2-21} & B_{2.22}
\end{array}\right]
$$$$
\times\left[\begin{array}{ll}
B_{2 \_11} & B_{2 \_12} \\
B_{2 \_1} & B_{2 \_22}
\end{array}\right]^{T}\left[\begin{array}{cc}
P_{1} & 0 \\
0 & P_{2}
\end{array}\right]
$$$$
\left.+\left[\begin{array}{cc}
P_{1} & 0 \\
0 & P_{2}
\end{array}\right]\right) X(t)
$$

$$
<\left\|x_{1}\right\|^{2}\left\{\alpha _ { 1 } \left[-1+\beta P_{\lambda 1}^{2}\right.\right.
$$

$$
\begin{gathered}
\times\left(\left\|B_{1 \_11}\right\|^{2}+\left\|B_{1 \_12}\right\|^{2}\right)+P_{\lambda 1}+P_{\lambda 1} \\
\times\left\|A_{1 \_12}\right\|+P_{\lambda 2}\left\|A_{1 \_1}\right\|+\beta P_{\lambda 1} P_{\lambda 2} \\
\left.\times\left(\left\|B_{1 \_11}\right\|\left\|B_{1 \_1}\right\|+\left\|B_{1 \_12}\right\|\left\|B_{1 \_22}\right\|\right)\right] \\
+\alpha_{2}\left[2 P_{\lambda 1}\left\|A_{2 \_11}\right\|+\beta P_{\lambda 1}^{2}\right. \\
\times\left(\left\|B_{2 \_11}\right\|^{2}+\left\|B_{2 \_12}\right\|^{2}\right)+P_{\lambda 1} \\
+P_{\lambda 2}\left\|A_{2 \_21}\right\|+P_{\lambda 1}\left\|A_{2 \_12}\right\| \\
+\beta P_{\lambda 1} P_{\lambda 2}\left(\left\|B_{2 \_11}\right\|\left\|B_{2 \_21}\right\|\right. \\
\left.\left.\left.+\left\|B_{2 \_12}\right\|\left\|B_{2 \_22}\right\|\right)\right]\right\}
\end{gathered}
$$

$$
\begin{aligned}
+\left\|x_{2}\right\|^{2}\left\{\alpha _ { 2 } \left[-1+\beta P_{\lambda 2}^{2}\right.\right. \\
\times\left(\left\|B_{2.21}\right\|^{2}+\left\|B_{2.22}\right\|^{2}\right)+P_{\lambda 2}
\end{aligned}
$$




$$
\begin{aligned}
+ & P_{\lambda 2}\left\|A_{2 \_21}\right\|+P_{\lambda 1}\left\|A_{2 \_12}\right\| \\
+ & \beta P_{\lambda 1} P_{\lambda 2}\left(\left\|B_{2 \_11}\right\|\left\|B_{2 \_21}\right\|\right. \\
& \left.\left.+\left\|B_{2 \_12}\right\|\left\|B_{2 \_22}\right\|\right)\right] \\
+\alpha_{1} & {\left[2 P_{\lambda 2}\left\|A_{1 \_22}\right\|+\beta P_{\lambda 2}^{2}\right.} \\
\times & \left(\left\|B_{1 \_21}\right\|^{2}+\left\|B_{1 \_22}\right\|^{2}\right)+P_{\lambda 2}+P_{\lambda 1} \\
\times & \left\|A_{1 \_12}\right\|+P_{\lambda 2}\left\|A_{1 \_1}\right\|+\beta P_{\lambda 1} P_{\lambda 2} \\
\times & \left(\left\|B_{1 \_11}\right\|\left\|B_{1 \_21}\right\|\right. \\
& \left.\left.\left.+\left\|B_{1 \_12}\right\|\left\|B_{1 \_2}\right\|\right)\right]\right\} .
\end{aligned}
$$

Furthermore, let $\alpha_{1}=\alpha, \alpha_{2}=1-\alpha$; therefore,

$$
\begin{aligned}
\dot{V}(X(t))< & \left\|x_{1}\right\|^{2}\left[\alpha S_{1 \_1}+(1-\alpha) S_{1 \_2}\right] \\
& +\left\|x_{2}\right\|^{2}\left[(1-\alpha) S_{2 \_}+\alpha S_{1 \_1}\right]
\end{aligned}
$$

If inequalities ((16a), (16b) and, (16c)) are satisfied and constant $\alpha \in(\underline{\alpha}, \bar{\alpha})$, then $\dot{V}(X(t))<0$. Hence, there exists a switching law such that system (1a) and (1b) is asymptotically stable.

Switching Law. Switched large-scale time-delay system ((1a), (1b)) with $r=N=2$ is switched to or stay at mode $i$ at time $t$ if inequality (23) is satisfied at time $t$ :

$$
\begin{aligned}
\sum_{i=1}^{2} x_{i}^{T}(t) P_{i}\left(A_{l-i i} x_{i}(t)+\sum_{\substack{j \neq i \\
j=1}}^{2} A_{l i j} x_{j}(t)\right. \\
\left.+\sum_{j=1}^{2} B_{l i j} x_{j}(t-\tau)\right)<0 \quad l=1,2 .
\end{aligned}
$$

Furthermore, we can extend to the case of $r=N$ switched systems. In the light of Lemma 2 , it is obvious that the stability of the switched large-scale time-delay system (1a) and (1b) is equivalent to that of the system

$$
\dot{X}(t)=\sum_{l=1}^{N} \alpha_{l}\left(A_{l} X(t)+B_{l} X(t-\tau)\right) .
$$

To investigate the stability of system (24), we choose the Lyapunov function candidate as

$$
V(X(t))=X^{T}(t) P X(t),
$$

where $P=\operatorname{diag}\left(P_{1}, P_{2}, \ldots, P_{N}\right)$ are unique real symmetric positive-definite matrices satisfying the Lyapunov equations

$$
A_{i j i}^{T} P_{i}+P_{i} A_{i j i}=-I, \quad 1 \leq i \leq N .
$$

Without loss of generality, $A_{i, i}$ are Hurwitz matrices.
Now, another sufficient condition for the stabilization of switched large-scale time-delay system (1a) and (1b) with $r=$ $N$ switched subsystems is addressed as the following theorem.

Theorem 6. Suppose the switched large-scale time-delay system (1a) and (1b) with $N$ switched subsystems, and then there exists a switching law such that switched large-scale timedelay system (1a) and (1b) is asymptotically stable of delay independence if constants $\alpha_{l}$ satisfy

$$
\sum_{l=1}^{N} \alpha_{l}=1
$$

$$
\begin{aligned}
& \alpha_{l}\left[-1+P_{\lambda l}^{2} \sum_{1 \leq i \leq N}\left\|B_{l\lrcorner i}\right\|^{2}+P_{\lambda l}+P_{\lambda l} \sum_{\substack{1 \leq j \leq N \\
j \neq l}}\left\|A_{l-l j}\right\|\right. \\
& \left.+P_{\lambda l} \sum_{\substack{1 \leq i \leq N \\
i \neq l}} P_{\lambda i}\left(\sum_{1 \leq j \leq N}\left\|B_{l\lrcorner l j}\right\|\left\|B_{l i j}\right\|\right)\right] \\
& +\sum_{\substack{1 \leq j \leq N \\
j \neq l}} \alpha_{j}\left[2 P_{\lambda i}\left\|A_{j i i}\right\|+P_{\lambda l}^{2} \sum_{1 \leq i \leq N}\left\|B_{j\lrcorner l}\right\|^{2}+P_{\lambda l}+P_{\lambda j}\right. \\
& \times\left\|A_{j-j l}\right\|+P_{\lambda l} \sum_{\substack{1 \leq i \leq N \\
i \neq l}} P_{\lambda i}\left(\sum_{1 \leq k \leq N}\left\|B_{j\lrcorner k}\right\|\left\|B_{j i k}\right\|\right) \\
& \left.+\sum_{\substack{1 \leq i \leq N \\
i \neq j}} P_{\lambda i} \sum_{\substack{1 \leq k \leq N \\
k \neq i \\
k \neq j}}\left\|A_{j \_i k}\right\|\right]<0 .
\end{aligned}
$$

Proof. With the same as proof of Theorem 5, Theorem 6 can be proved.

Switching Law. The switched time-delay system (1a) and (1b) is switched to or stay at mode $i$ at time $t$ if inequality (28) is satisfied at time $t$ :

$$
\begin{array}{r}
\sum_{i=1}^{N} x_{i}^{T}(t) P_{i}\left(A_{l i i} x_{i}(t)+\sum_{\substack{j \neq i \\
j=1}}^{N} A_{l \_i j} x_{j}(t)\right. \\
\left.+\sum_{j=1}^{N} B_{i j} x_{j}(t-\tau)\right)<0, \quad l=1,2, \ldots, N .
\end{array}
$$

Corollary 7. The nominal large-scale switched systems (1a) and (1b) without time-delay, that is, $B_{l i j}=0$, and then there 
exists a switching law such that switched large-scale system is asymptotically stable if constants $\alpha_{l}$ satisfy

$$
\begin{aligned}
& \sum_{l=1}^{N} \alpha_{l}=1 \\
& \alpha_{l}\left(-1+P_{\lambda l} \sum_{\substack{j \neq l \\
j=1}}^{N}\left\|A_{l l j}\right\|\right) \\
& +\sum_{\substack{j=1 \\
j \neq l}}^{N} \alpha_{j}\left(2 P_{\lambda l}\left\|A_{j l l}\right\|+P_{\lambda_{j}}\left\|A_{j_{-} j l}\right\|+\right. \\
& \left.+\sum_{\substack{i=1 \\
i \neq j}}^{N} P_{\lambda i} \sum_{\substack{k=1 \\
k \neq i \\
k \neq j}}^{N}\left\|A_{j i k}\right\|\right)<0 \\
& \text { for } l=1,2, \ldots, N \text {. }
\end{aligned}
$$

Furthermore, we can develop another method to investigate the stability of switched large-scale time-delay systems (1a) and (1b).

In the light of Lemma 1, it is obvious that the stability of the switched time-delay system (1a) and (1b) is equivalent to that of the switched large-scale system

$$
\dot{w}_{i}(t)=\bar{A}_{l_{-} i i} w_{i}(t)+\sum_{\substack{j \neq i \\ j=1}}^{N} \bar{A}_{l_{-i j}} w_{j}(t), \quad \forall|z| \leq 1,
$$

where $\bar{A}_{l_{-i i}}=A_{l_{-i i}}+z B_{l_{-i i}}$ and $\bar{A}_{l_{-i j}}=A_{l_{i j}}+z B_{l_{-i j}}$.

In the light of Lemma 4 , a convex combination of switched large-scale systems (31) can be written as follows:

$$
\dot{w}_{i}(t)=\sum_{l=1}^{r} \alpha_{l}\left[\left(\bar{A}_{l_{-} i i} w_{i}(t)+\sum_{\substack{j \neq i \\ j=1}}^{N} \bar{A}_{l_{i j}} w_{j}(t)\right)\right] .
$$

First, we consider the switched system (la) and (1b) with $r=N=2$. In the light of Lemma 4 , it is obvious that the stability of the switched time-delay system is equivalent to that of the system

$$
\begin{aligned}
\dot{W}(t)= & {\left[\alpha\left(A_{1}+z B_{1}\right)+(1-\alpha)\left(A_{2}+z B_{2}\right)\right] W(t) } \\
= & \alpha\left[\begin{array}{ll}
A_{1 \_11}+z B_{1 \_11} & A_{1 \_12}+z B_{1 \_12} \\
A_{1 \_21}+z B_{1 \_21} & A_{1 \_22}+z B_{1 \_22}
\end{array}\right]+(1-\alpha) \\
& \left.\times\left[\begin{array}{ll}
A_{2 \_11}+z B_{2 \_11} & A_{2 \_12}+z B_{2 \_12} \\
A_{2 \_21}+z B_{2 \_11} & A_{2 \_22}+z B_{2 \_22}
\end{array}\right]\right) W(t),
\end{aligned}
$$

where $0 \leq \alpha \leq 1$
To investigate the stability of system (33), we choose the Lyapunov function candidate as

$$
V_{i}\left(w_{i}(t)\right)=w_{i}^{T} P_{i} w_{i}
$$

where $W(t)=\left[w_{1}^{T} w_{2}^{T}\right]^{T}$ and $P=\operatorname{diag}\left[P_{1}, P_{2}\right]$ are unique real symmetric positive-definite matrices satisfying the Lyapunov equations (14).

It will be convenient throughout the remainder of this section to use the following notations:

$$
\begin{aligned}
& f_{2 \_11}=\left\|A_{2 \_11}\right\|+\left\|B_{2 \_11}\right\|, \quad f_{1 \_22}=\left\|A_{1 \_22}\right\|+\left\|B_{1 \_22}\right\|, \\
& f_{1 \_12}=\left\|A_{1 \_12}\right\|+\left\|B_{1 \_12}\right\|, \quad f_{2 \_21}=\left\|A_{2 \_21}\right\|+\left\|B_{2 \_11}\right\| \text {, } \\
& f_{2 \_12}=\left\|A_{2 \_12}\right\|+\left\|B_{2 \_12}\right\|, \quad f_{1 \_21}=\left\|A_{1 \_21}\right\|+\left\|B_{1 \_21}\right\| \text {, } \\
& P_{\lambda 1}=\operatorname{Max}\left[\lambda\left(P_{1}\right)\right], \quad P_{\lambda 2}=\operatorname{Max}\left[\lambda\left(P_{2}\right)\right], \\
& \underline{\underline{\alpha}}=\left[\left(2 f_{2 \_11}+f_{2 \_12}\right) P_{\lambda 1}+f_{2 \_1} P_{\lambda 2}\right] \\
& \times\left(\left(2 f_{2 \_11}+f_{2 \_12}\right) P_{\lambda 1}+f_{2 \_21} P_{\lambda 2}\right. \\
& \left.+1-\left[\left(2\left\|B_{1 \_11}\right\|+f_{1 \_12}\right) P_{\lambda 1}+f_{1 \_1} P_{\lambda 2}\right]\right)^{-1}, \\
& \overline{\bar{\alpha}}=\left(1-\left[\left(2\left\|B_{2.22}\right\|+f_{2 \_1}\right) P_{\lambda 2}+f_{2 \_12} P_{\lambda 1}\right]\right) \\
& \times\left(\left(2 f_{1 \_22}+f_{1 \_21}\right) P_{\lambda 2}+f_{1 \_12} P_{\lambda 1}+1\right. \\
& \left.-\left[\left(2\left\|B_{2 \_22}\right\|+f_{2 \_1}\right) P_{\lambda 2}+f_{2 \_12} P_{\lambda 1}\right]\right)^{-1} .
\end{aligned}
$$

Now, another sufficient condition for the stabilization of switched large-scale time-delay system (la) and (1b) with $r=$ $N=2$ is addressed as the following theorem.

Theorem 8. Suppose the switched time-delay system (1a) and (1b) with $r=N=2$. These exists a switching law such that the switched time-delay system (1a) and (1b) is asymptotically stable of delay independence if constant $\alpha \in(\underline{\underline{\alpha}}, \overline{\bar{\alpha}})$ and there matrices $P_{1}, P_{2}>0$ satisfying

$$
\begin{aligned}
\text { (i) } 1- & {\left[\left(2\left\|B_{1 \_11}\right\|+f_{1 \_12}\right) P_{\lambda 1}+f_{1 \_21} P_{\lambda 2}\right]>0 } \\
\text { (ii) } 1- & {\left[\left(2\left\|B_{2 \_22}\right\|+f_{2 \_21}\right) P_{\lambda 2}+f_{2 \_12} P_{\lambda 1}\right]>0 } \\
\text { (iii) }\{1- & {\left.\left[\left(2\left\|B_{1 \_11}\right\|+f_{1 \_12}\right) P_{\lambda 1}+f_{1 \_21} P_{\lambda 2}\right]\right\} } \\
\times & \left\{1-\left[\left(2\left\|B_{2 \_2}\right\|+f_{2 \_21}\right) P_{\lambda 2}+f_{2 \_12} P_{\lambda 1}\right]\right\} \\
> & {\left[\left(2 f_{2 \_11}+f_{2 \_12}\right) P_{\lambda 1}+f_{2 \_1} P_{\lambda 2}\right] } \\
\times & {\left[\left(2 f_{1 \_22}+f_{1 \_21}\right) P_{\lambda 2}+f_{1 \_12} P_{\lambda 1}\right] . }
\end{aligned}
$$

Proof. The Lyapunov function for the overall system is

$$
V(W(t))=\sum_{i=1}^{2} w_{i}^{T}(t) P_{i} w_{i}(t)=W^{T}(t) P W(t) .
$$


The differential of the Lyapunov function (37) is as follows

$$
\begin{aligned}
& \dot{V}(W(t))=\sum_{i=1}^{2}\left[\dot{w}_{i}^{T}(t) P_{i} w_{i}(t)+w_{i}^{T}(t) P_{i} \dot{w}_{i}(t)\right] \\
& =\dot{W}^{T}(t) P W(t)+W^{T} P \dot{W} \\
& \leq\left\|w_{1}\right\|^{2}\left\{2 \left[\alpha\left(\left\|A_{1 \_11}\right\|+\left\|B_{1 \_11}\right\|\right)\right.\right. \\
& \left.+(1-\alpha) f_{2 \_11}\right] P_{\lambda 1} \\
& +\frac{1}{2}\left[\left(\alpha f_{1 \_12}+(1-\alpha) f_{2 \_12}\right) P_{\lambda 1}\right. \\
& \left.\left.+\left(\alpha f_{1 \_21}+(1-\alpha) f_{2 \_21}\right) P_{\lambda 2}\right]\right\} \\
& +\frac{1}{2}\left\|w_{2}\right\|^{2}\left[\left(\alpha f_{1-12}+(1-\alpha) f_{2-12}\right) P_{\lambda_{1}}\right. \\
& \left.+\left(\alpha f_{1 \_21}+(1-\alpha) f_{2.21}\right) P_{\lambda 2}\right] \\
& +\left\|w_{2}\right\|^{2}\left\{2 \left[f_{1.22}+(1-\alpha)\right.\right. \\
& \left.\times\left(\left\|A_{2 \_22}\right\|+\left\|B_{2 \_22}\right\|\right)\right] P_{\lambda 2} \\
& +\frac{1}{2}\left[\left(\alpha f_{1 \_12}+(1-\alpha) f_{2 \_12}\right) P_{\lambda 1}\right. \\
& \left.\left.+\left(\alpha f_{121}+(1-\alpha) f_{2.21}\right) P_{\lambda 2}\right]\right\} \\
& +\frac{1}{2}\left\|w_{1}\right\|^{2}\left[\left(\alpha f_{1 \_12}+(1-\alpha) f_{2 \_12}\right) P_{\lambda 1}\right. \\
& \left.+\left(\alpha f_{1 \_21}+(1-\alpha) f_{2 \_21}\right) P_{\lambda 2}\right] \\
& \leq\left\|w_{1}\right\|^{2}\left\{-\alpha\left[1-\left[\left(2\left\|B_{1 \_11}\right\|+f_{1 \_12}\right) P_{\lambda 1}\right.\right.\right. \\
& \left.\left.+f_{1.21} P_{\lambda 2}\right]\right]+(1-\alpha) \\
& \left.\times\left[\left(2 f_{2 \_11}+f_{2 \_12}\right) P_{\lambda 1}+f_{2 \_21} P_{\lambda 2}\right]\right\} \\
& +\left\|w_{2}\right\|^{2}\left\{( \alpha - 1 ) \left[1-\left[\left(2\left\|B_{222}\right\|+f_{221}\right) P_{\lambda 2}\right.\right.\right. \\
& \left.\left.+f_{2-12} P_{\lambda 1}\right]\right] \\
& \left.+\alpha\left[\left(2 f_{1.22}+f_{1.21}\right) P_{\lambda 2}+f_{1 \_12} P_{\lambda 1}\right]\right\} \text {. }
\end{aligned}
$$

If inequalities ((36a), (36b), and (36c)) are satisfying and constant $\alpha \in(\underline{\underline{\alpha}}, \overline{\bar{\alpha}})$, then $\dot{V}(W(t))<0$. Hence, there exists a switching law such that system (la) and (1b) is asymptotically stable.

Switching Law. Switched time-delay system (1a) and (1b) with $r=N=2$ is switched to or stay at mode $i$ at time $t$ if inequality (39) is satisfied at time $t$ :

$$
\begin{aligned}
\sum_{i=1}^{2} x_{i}^{T}(t) P_{i}\left(A_{l i i} x_{i}(t)+\sum_{\substack{j \neq i \\
j=1}}^{2} A_{l \_i j} x_{j}(t)\right. \\
\left.+\sum_{j=1}^{2} B_{l-i j} x_{j}(t-\tau)\right)<0 \quad l=1,2 .
\end{aligned}
$$

Now, a sufficient condition for the stabilization of switched large-scale time-delay system (la) and (1b) with $r=$ $N$ switched systems is addressed as the following theorem.

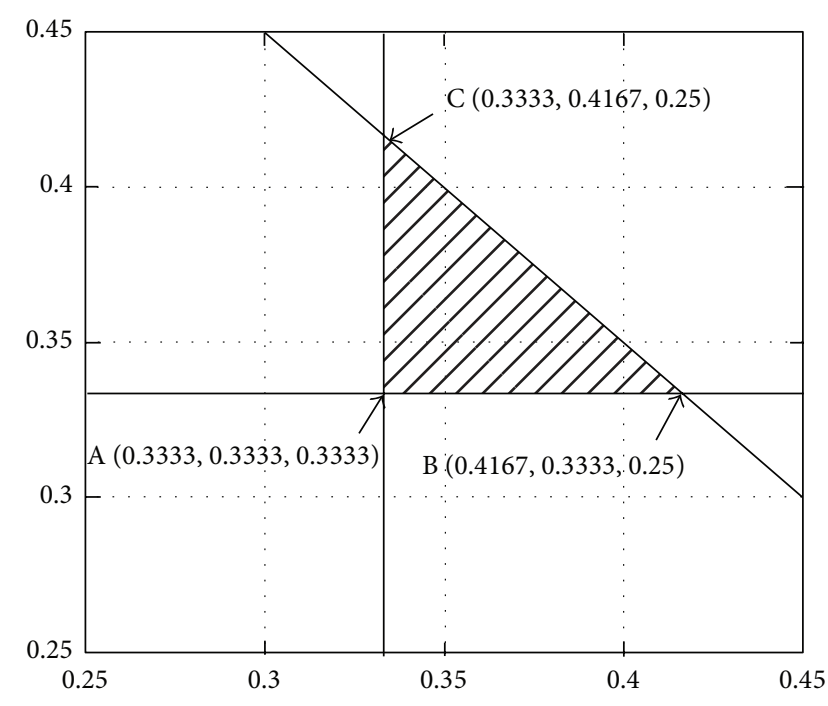

Figure 1: The admissible region $\alpha_{1}$ and $\alpha_{2}$ (x label: $\alpha_{1}, y$ label: $\alpha_{2}$ and $\left.\alpha_{1}+\alpha_{2}+\alpha_{3}=1\right)$.

Theorem 9. Suppose the switched large-scale time-delay system (1a) and (1b) with arbitrary $N$ switched systems, and then there exists a switching law such that switched large-scale timedelay system (1a) and (1b) is asymptotically stable of delay independence if constants $\alpha_{l}$ satisfy

$$
\begin{gathered}
\sum_{l=1}^{N} \alpha_{l}=1, \\
-\alpha_{l}\left\{1-\left[2\left\|B_{l\lrcorner l}\right\| P_{\lambda l}+\sum_{\substack{i \neq l \\
i=1}}^{N} f_{l\lrcorner i} P_{\lambda l}+\sum_{\substack{i \neq l \\
i=1}}^{N} f_{l \_l l} P_{\lambda i}\right]\right\} \\
+\sum_{\substack{j \neq l \\
j=1}}^{N} \alpha_{j}\left(f_{j \_l j} P_{\lambda l}+\sum_{\substack{i \neq j \\
i=1}}^{N} f_{j\lrcorner i} P_{\lambda l}+\sum_{i=1}^{N} f_{j \_l l} P_{\lambda i}\right)<0,
\end{gathered}
$$

where $f_{l \perp i}=\left\|A_{l\lrcorner i}\right\|+\left\|B_{l\lrcorner i}\right\|, \quad f_{j \_i}=\left\|A_{j\lrcorner i}\right\|+\left\|B_{j \_i}\right\|, \quad f_{j . i l}=$

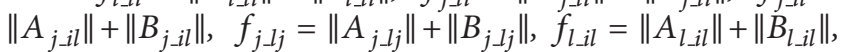
and $\lambda_{\text {max }}\left(P_{i}\right)=P_{\lambda i}, i=1,2, \ldots, N$.

Proof. With the same as proof of Theorem 8, Theorem 9 can be proved.

Corollary 10. The nominal switched large-scale systems (1a) and (1b) without time-delay, that is, $B_{l i j}=0$, and then there exists a switching law such that switched large-scale system is asymptotically stable if constants $\alpha_{l}$ satisfy

$$
\sum_{l=1}^{N} \alpha_{l}=1
$$




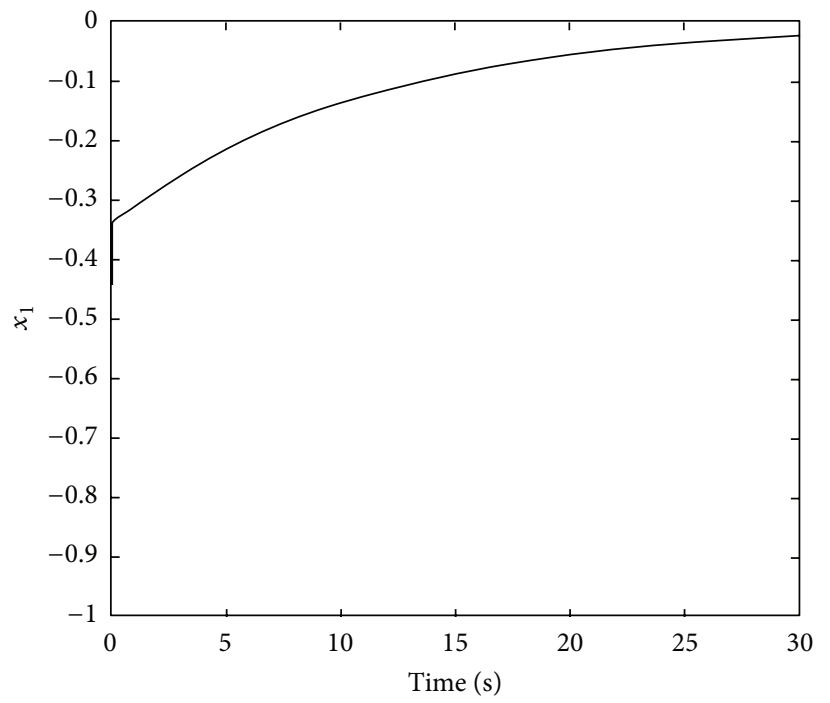

(a)

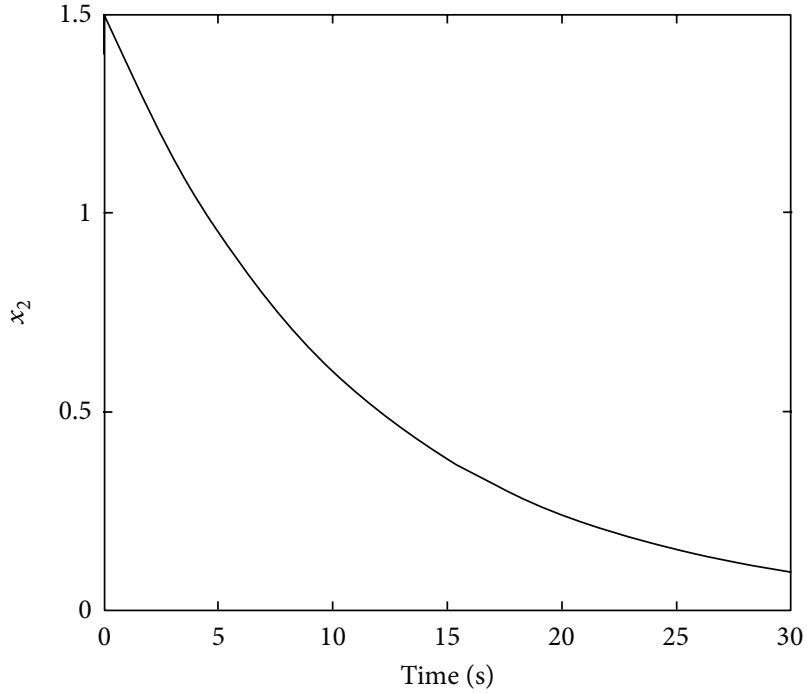

(b)

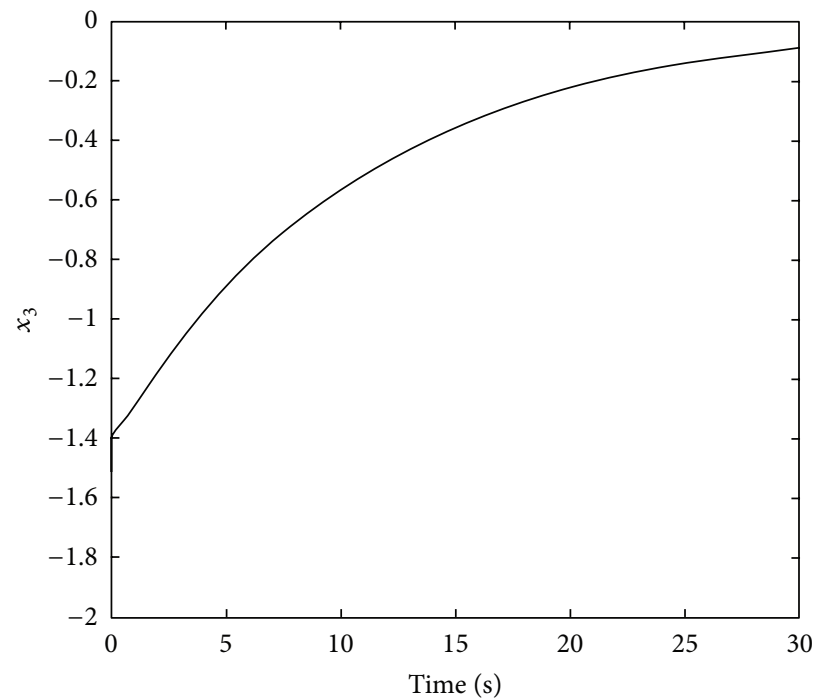

(c)

Figure 2: (a) State response for $x_{1}$. (b) State response for $x_{2}$. (c) State response for $x_{3}$.

$$
\begin{array}{r}
-\alpha_{l}\left[1-\left(\sum_{\substack{i \neq l \\
i=1}}^{N}\left\|A_{l \_l i}\right\| P_{\lambda l}+\sum_{\substack{i \neq l \\
i=1}}^{N}\left\|A_{l i l}\right\| P_{\lambda i}\right)\right] \\
+\sum_{\substack{j \neq l \\
j=1}}^{N} \alpha_{j}\left(\left\|A_{j\lrcorner j}\right\| P_{\lambda l}+\sum_{\substack{i \neq j \\
i=1}}^{N}\left\|A_{j\lrcorner i}\right\| P_{\lambda l}\right. \\
\left.+\sum_{i=1}^{N}\left\|A_{j i l}\right\| P_{\lambda i}\right)<0 \quad l=1,2, \ldots, N .
\end{array}
$$

Remark 11. The main contribution of the results is that this method can be applied to cases when all individual switched systems are unstable in this paper.

\section{Numerical Example}

Example 12. Consider a switched time-delay system composed of three individual switched systems given as follow. Switched System 1:

$$
\begin{gathered}
\dot{x}_{1}(t)=-2.5 x_{1}(t)+0.1 x_{1}(t-\tau) \\
\dot{x}_{2}(t)=0.1 x_{2}(t)-0.12 x_{1}(t-\tau)-0.06 x_{2}(t-\tau) \\
+0.08 x_{3}(t-\tau) \\
\dot{x}_{3}(t)=0.2 x_{3}(t)+0.06 x_{1}(t-\tau)+0.08 x_{2}(t-\tau) \\
+0.06 x_{3}(t-\tau) .
\end{gathered}
$$

Switched System 2:

$$
\begin{aligned}
\dot{x}_{1}(t)= & 0.1 x_{1}(t)+0.1 x_{1}(t-\tau)+0.02 x_{2}(t-\tau) \\
& +0.14 x_{3}(t-\tau)
\end{aligned}
$$




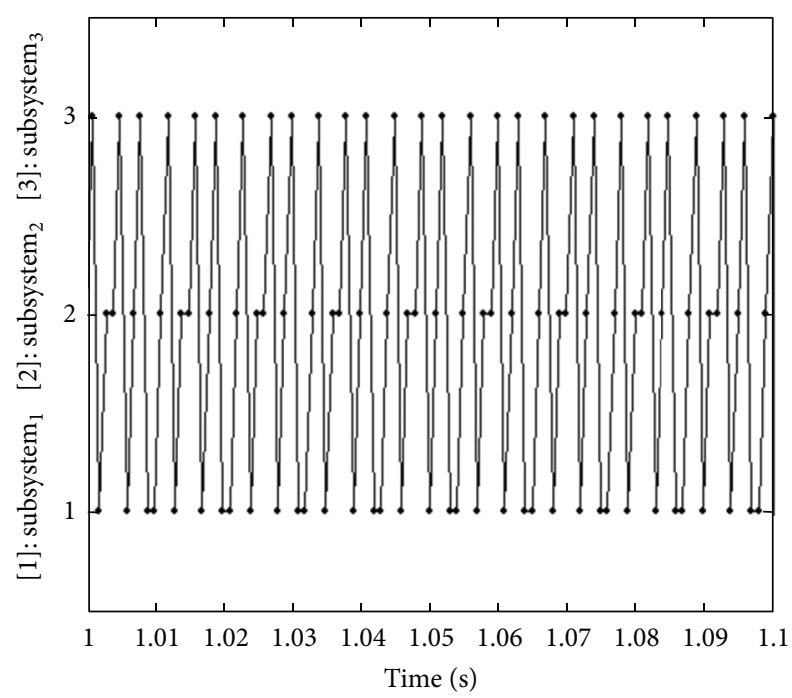

FIGURE 3: The switching times of the system during $[1,1.1] \mathrm{sec}$.

$$
\begin{aligned}
\dot{x}_{2}(t)= & -4 x_{2}(t)+0.04 x_{1}(t-\tau)+0.176 x_{2}(t-\tau) \\
& +0.132 x_{3}(t-\tau) \\
\dot{x}_{3}(t)= & 0.2 x_{3}(t)-0.02 x_{1}(t-\tau)-0.068 x_{2}(t-\tau) \\
& -0.176 x_{3}(t-\tau) .
\end{aligned}
$$

Switched System 3:

$$
\begin{aligned}
\dot{x}_{1}(t)= & 0.2 x_{1}(t)+0.08 x_{1}(t-\tau)-0.1 x_{2}(t-\tau) \\
\dot{x}_{2}(t)= & 0.2 x_{2}(t)+0.044 x_{1}(t-\tau)+0.18 x_{2}(t-\tau) \\
& +0.02 x_{3}(t-\tau) \\
\dot{x}_{3}(t)= & -3 x_{3}(t)-0.092 x_{1}(t-\tau)-0.04 x_{2}(t-\tau) \\
& +0.04 x_{3}(t-\tau) .
\end{aligned}
$$

Via normal tests of stability for the large-scale time-delay system, three individual switched systems ((42a), (42b), and (42c)) are all unstable. Furthermore, according to Lyapunov equation (26), the $P_{i}(i=1,2,3)$ can be obtained as the following:

$$
P_{1}=0.2, \quad P_{2}=0.125, \quad P_{3}=0.1667 \text {. }
$$

In view of the stability conditions of Theorem 6 , the conditions (27a) and (27b) can be written as follows:

$$
\begin{gathered}
\text { (i) } \alpha_{1}+\alpha_{2}+\alpha_{3}=1 \\
\text { (ii) }-0.7874 \alpha_{1}+0.2472 \alpha_{2}+0.3023 \alpha_{3}<0 \\
\text { (iii) } 0.1607 \alpha_{1}-0.8668 \alpha_{2}+0.1924 \alpha_{3}<0 \\
\text { (iv) } 0.2410 \alpha_{1}+0.2408 \alpha_{2}-0.8119 \alpha_{3}<0 .
\end{gathered}
$$

Furthermore, let $\alpha_{3}=1-\alpha_{1}-\alpha_{2}$; the above conditions ((44a), (44b), and (44c)) can be integrated as

$$
\begin{aligned}
& -1.0897 \alpha_{1}-0.0551 \alpha_{2}+0.3023<0 \\
& -0.0318 \alpha_{1}-1.0592 \alpha_{2}+0.1924<0 \\
& 1.0528 \alpha_{1}+1.0526 \alpha_{2}-0.8119<0 .
\end{aligned}
$$

Therefore, $\alpha_{1}$ and $\alpha_{2}$ are selected inside the admissible region of the triangle as shown in Figure 1, and the switched timedelay system $((42 a),(42 b)$, and $(42 c))$ is asymptotically stable by the following switching law:

$$
\begin{aligned}
\sum_{i=1}^{3} x_{i}^{T}(t) P_{i}\left(A_{l i i} x_{i}(t)+\sum_{\substack{j \neq i \\
j=1}}^{3} A_{l_{-i j}} x_{j}(t)\right. \\
\left.\quad+\sum_{j=1}^{3} B_{l i j} x_{j}(t-\tau)\right)<0, \quad l=1,2,3 .
\end{aligned}
$$

In view of the stability conditions of Theorem 9, in addition, the conditions (40a) and (40b) can be written as follows:

$$
\text { (i) } \alpha_{1}+\alpha_{2}+\alpha_{3}=1
$$

$$
\text { (ii) }-0.9350 \alpha_{1}+0.1203 \alpha_{2}+0.1528 \alpha_{3}<0
$$

(iii) $0.0783 \alpha_{1}-0.9192 \alpha_{2}+0.1297 \alpha_{3}<0$

(iv) $0.1200 \alpha_{1}+0.1845 \alpha_{2}-0.9622 \alpha_{3}<0$.

Furthermore, let $\alpha_{3}=1-\alpha_{1}-\alpha_{2}$; the above conditions ((47a), $(47 \mathrm{~b})$, and $(47 \mathrm{c}))$ can be integrated as

$$
\begin{aligned}
& -1.0878 \alpha_{1}-0.0325 \alpha_{2}+0.1528<0 \\
& -0.0513 \alpha_{1}-1.0488 \alpha_{2}+0.1297<0 \\
& 1.0822 \alpha_{1}+1.1467 \alpha_{2}-0.9622<0 .
\end{aligned}
$$

Therefore, $\alpha_{1}$ and $\alpha_{2}$ are selected inside the admissible region of the triangle as shown in Figure 2.

The trajectories of the switched large-scale time-delay system ((42a), (42b), and (42c)) and the switching during period $[0.5,0.6] \mathrm{sec}$ are shown in Figure 3 with initial value $X(0)=\left[\begin{array}{lll}2 & -1 & 3\end{array}\right]^{T}$ and $\tau=0.5 \mathrm{sec}$.

\section{Conclusion}

We have shown that the Lyapunov stability theorem can deal with the stabilization of switched large-scale time-delay systems. In addition, it is shown that the main advantages of our approach are that it can be applied to cases when all individual switched systems are unstable, quantify the region of stability, extend to arbitrary subsystems of switched largescale time-delay systems, and develop the simple switching 
rule to stabilize the switched large-scale time-delay systems. For future research, we are interested in topics such as the stability problems of designing the switched laws with time dependency for the switched large-scale time-delay systems?

\section{Acknowledgment}

This work is supported by the National Science Council, Taiwan, under Grand nos. NSC 102-2221-E-218 -017 and NSC100-2632-E-218-001-MY3.

\section{References}

[1] A. S. Morse, "Supervisory control of families of linear setpoint controllers-part I: exact matching," IEEE Transactions on Automatic Control, vol. 41, no. 10, pp. 1413-1431, 1996.

[2] D. Liberzon and A. S. Morse, "Basic problems in stability and design of switched systems," IEEE Control Systems Magazine, vol. 19, no. 5, pp. 59-70, 1999.

[3] H. Sun and L. Hou, "Composite disturbance observer-based control and $H_{\infty}$ output tracking control for discrete-time switched systems with time-varying delay," Mathematical Problems in Engineering, vol. 2013, Article ID 698935, 12 pages, 2013.

[4] J. P. Hespanha and A. S. Morse, "Stability of switched systems with average dwell-time," in Proceedings of the 38th IEEE Conference on Decision and Control (CDC '99), pp. 2655-2660, December 1999.

[5] G. Zhai, H. Lin, and P. J. Antsaklis, "Quadratic stabilizability of switched linear systems with polytopic uncertainties," International Journal of Control, vol. 76, no. 7, pp. 747-753, 2003.

[6] Z. Li, Y. Soh, and C. Wen, Switched and Impulsive Systems: Analysis, Design and Application, Springer, Berlin, Germany, 2005.

[7] X. Bu, F. Yu, Z. Fu, and F. Wang, "Stability analysis of highorder iterative learning control for a class of nonlinear switched systems," Abstract and Applied Analysis, vol. 2013, Article ID 684642, 13 pages, 2013.

[8] S. Huang, Z. Xiang, and H. R. Karimi, "Stabilization and controller design of $2 \mathrm{D}$ discrete switched systems with state delays under asynchronous switching," Abstract and Applied Analysis, vol. 2013, Article ID 961870, 12 pages, 2013.

[9] Z. Dombovari, D. A. W. Barton, R. Eddie Wilson, and G. Stepan, "On the global dynamics of chatter in the orthogonal cuttingmodel," International Journal of Non-Linear Mechanics, vol. 46, no. 1, pp. 330-338, 2011.

[10] R. Szalai, G. Stepan, and S. J. Hogan, "Global dynamics of low immersion high-speed milling," Chaos, vol. 14, no. 4, pp. 10691077, 2004.

[11] J. Wei, P. Shi, H. R. Karimi, and B. Wang, "BIBO stability analysis for delay switched systems with nonlinear perturbation," Abstract and Applied Analysis, vol. 2013, Article ID 738653, 8 pages, 2013.

[12] Y. Zhang, Y. Zhang, J. Li, and B. Zhu, "Dissipative output tracking control of linear systems with time delay," Abstract and Applied Analysis, vol. 2013, Article ID 324741, 8 pages, 2013.

[13] G. Zhai, Y. Sun, X. Chen, and A. N. Michel, "Stability and $L_{2}$ gain analysis for switched symmetric systems with time delay," in Proceedings of the American Control Conference, pp. 26822687, Denver, Colo, USA, June 2003.

[14] J. Shi, T. Wu, and S. Du, "Delay-dependent robust $\mathrm{H}_{\infty}$ control for switched systems with parameter uncertainties and time delay," in Proceedings of the 5th World Congress on Intelligent Control and Automation, pp. 951-955, Hangzhou, China, June 2004.

[15] S. Huang and Z. Xiang, "Robust $L_{\infty}$ reliable control for uncertain switched nonlinear systems with time delay under asynchronous switching," Applied Mathematics and Computation, vol. 222, pp. 658-670, 2013.

[16] S. Huang and Z. Xiang, "Delay-dependent stability for discrete 2D switched systems with state delays in the roesser model," Circuits, Systems, and Signal Processing, vol. 32, no. 6, pp. 28212837, 2013.

[17] Z. Xiang and S. Huang, "Stability analysis and stabilization of discrete-time 2D switched systems," Circuits, Systems, and Signal Processing, vol. 32, no. 1, pp. 401-414, 2013.

[18] G. Yu, S. Niu, J. Ma, and Y. Song, "An adaptive predictioncorrection method for solving large-scale nonlinear systems of monotone equations with applications," Abstract and Applied Analysis, vol. 2013, Article ID 619123, 13 pages, 2013.

[19] M. Farid, W. J. Leong, N. Malekmohammadi, and M. Mamat, "Scaled diagonal gradient-type method with extra update for large-scale unconstrained optimization," Abstract and Applied Analysis, vol. 2013, Article ID 532041, 5 pages, 2013.

[20] C.-J. Wang and J.-S. Chiou, "A stability condition with delaydependence for a class of switched large-scale time-delay systems," Journal of Applied Mathematics, vol. 2013, Article ID 360170, 7 pages, 2013.

[21] C.-J. Wang and J.-S. Chiou, "Stabilization analysis for the switched large-scale discrete-time systems via the state-driven switching," Discrete Dynamics in Nature and Society, vol. 2013, Article ID 630545, 5 pages, 2013.

[22] H. Wu, N. Li, K. Wang, G. Xu, and Q. Guo, "Global robust stability of switched interval neural networks with discrete and distributed time-varying delays of neural type," Mathematical Problems in Engineering, vol. 2012, Article ID 361871, 18 pages, 2012.

[23] G. Zhang, T. Li, and S. Fei, "Further stability criterion on delayed recurrent neural networks based on reciprocal convex technique," Mathematical Problems in Engineering, vol. 2012, Article ID 829037, 14 pages, 2012.

[24] Y. Dong, S. Mei, and X. Wang, "Novel stability criteria of nonlinear uncertain systems with time-varying delay," Abstract and Applied Analysis, vol. 2011, Article ID 969674, 16 pages, 2011.

[25] A. Banakar, "Lyapunov stability analysis of gradient descentlearning algorithm in network training," ISRN Applied Mathematics, vol. 2011, Article ID 145801, 12 pages, 2011.

[26] A. Hmamed, "Further results on the robust stability of uncertain time-delay systems," International Journal of Systems Science, vol. 22, no. 3, pp. 605-614, 1991.

[27] J. Hale, Theory of Functional Differential Equations, Springer, New York, NY, USA, 1977. 


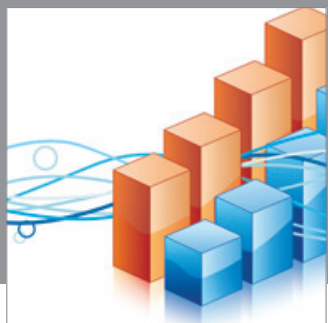

Advances in

Operations Research

mansans

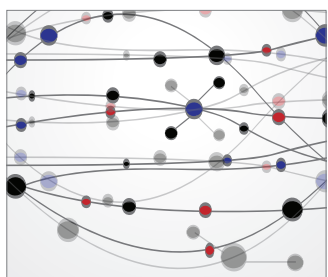

The Scientific World Journal
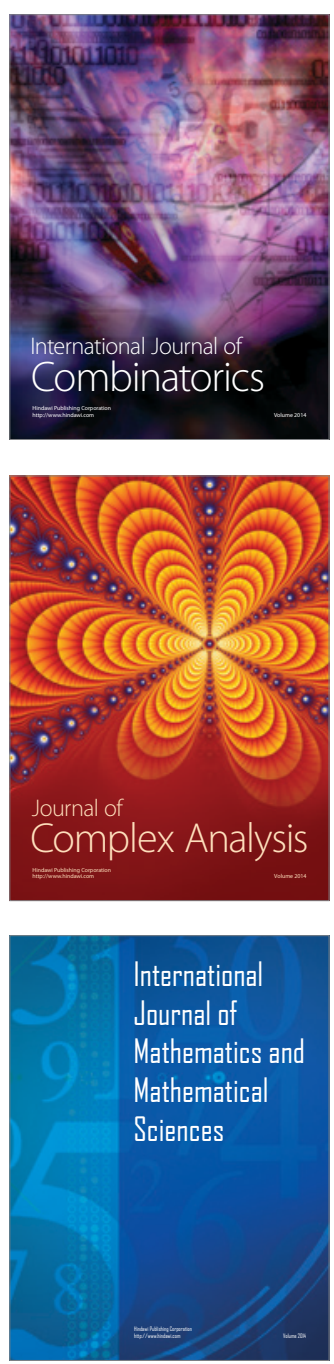
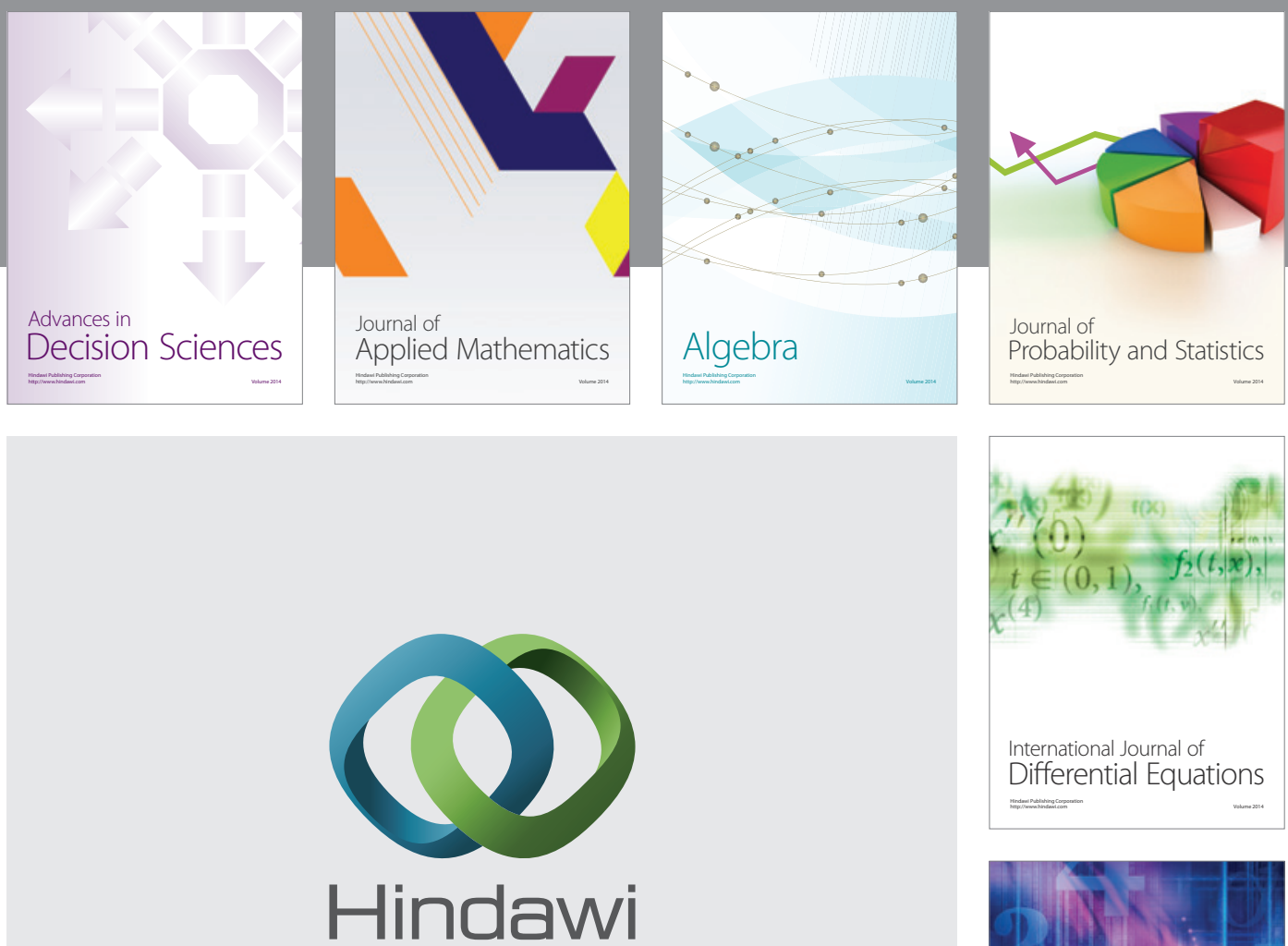

Submit your manuscripts at http://www.hindawi.com
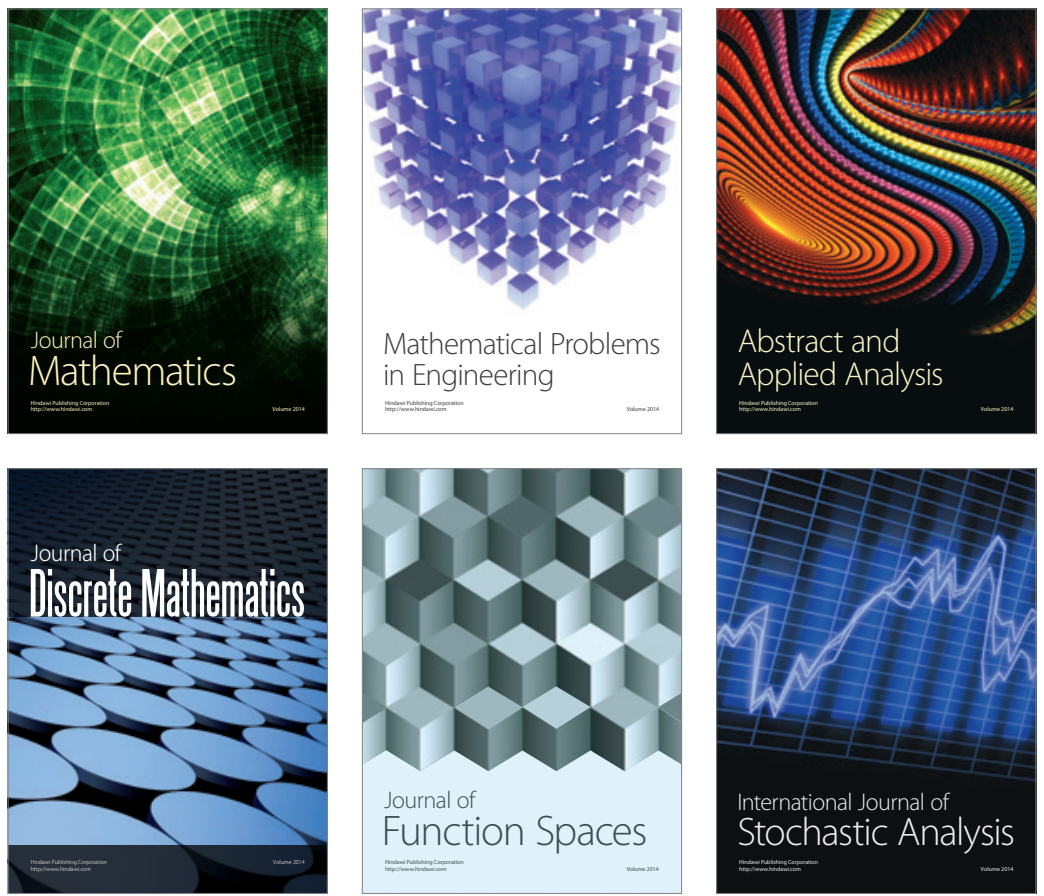

Journal of

Function Spaces

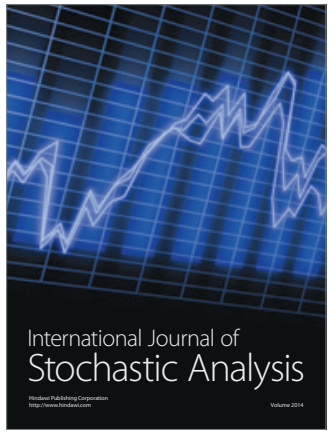

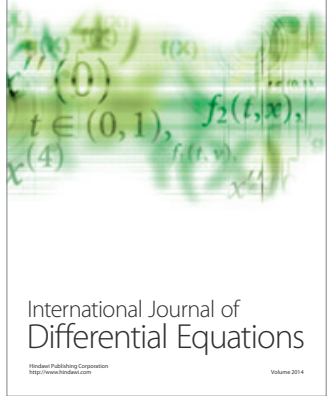
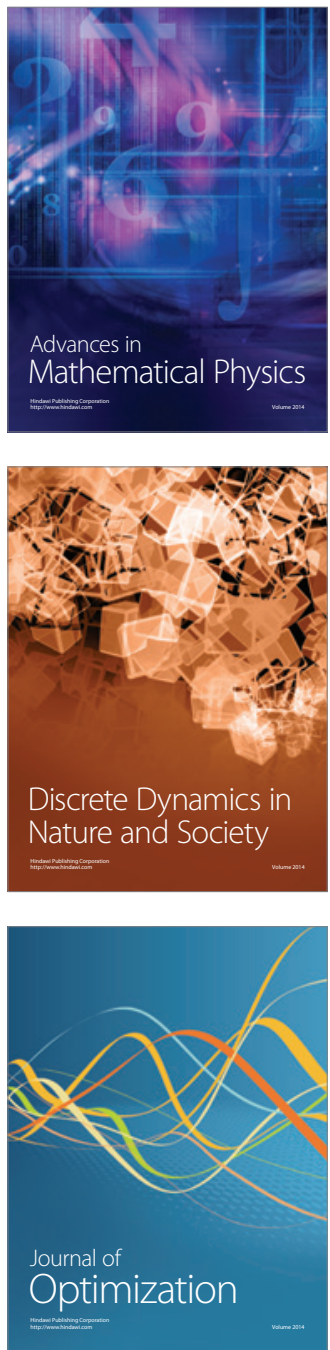